\title{
Clinical comparison of frequency doubling technology perimetry and Humphrey perimetry
}

\author{
Robert Casson, Bruce James, Adrian Rubinstein, Haggai Ali
}

\begin{abstract}
Aim-To determine the number of missed points on frequency doubling technology (FDT) perimetry that optimise the sensitivity and specificity of the test and to determine the topographical accuracy of the test in a clinical setting.

Methods-In a prospective study, the perimetric data from 99 patients who underwent both FDT perimetry in the screening mode and Humphrey 24-2 (H24-2) were used to determine the sensitivity and specificity of the FDT perimetry compared with the full threshold $\mathrm{H} 24-2$ as the gold standard.

Results-Missed points on the FDT perimetry correlated with both the mean deviation and the corrected pattern standard deviation on the Humphrey perimetry. A score assigned to abnormal points on the FDT perimetry and the Humphrey total deviation plot showed a significant correlation for both the location and the depth of the defect. In comparing the Humphrey hemifield test with the FDT perimetry results, if at least one missed point on the frequency doubling test was considered as abnormal then the overall sensitivity of the test was $78.1 \%$ and the specificity was $89.1 \%$.
\end{abstract}

Conclusion-FDT perimetry in the screening mode performed in a clinical setting was highly specific, exhibited reasonable sensitivity, and accurately determined the location and depth of scotomas when compared with the full threshold Humphrey 24-2.

(Br F Ophthalmol 2001;85:360-362)

Frequency doubling technology is a relatively new method of visual field testing that relies on an optical illusion first described by Kelly in 1966. ${ }^{1}$ The anatomical substrate of this effect has been ascribed to the retinal ganglion cells (RGCs), ${ }^{2}$ which are thought to be particularly susceptible to early glaucomatous damage. ${ }^{34}$ This has led to the development of frequency doubling technology (FDT) perimetry with particular use as a glaucoma screening tool. ${ }^{5-7}$ Preliminary results suggest that FDT perimetry is a rapid, valid test for glaucomatous field loss. ${ }^{8-16}$ In a clinical study of glaucoma suspects, Quigley ${ }^{8}$ found a sensitivity and specificity of over $90 \%$ compared with Humphrey full threshold perimetry. Other investigators have recently corroborated the high specificity but have reported a lower sensitivity. ${ }^{14-16}$

Although preliminary results are impressive, the optimal interpretation of FDT perimetry data is unclear. Further clinical data are needed before this test can be confidently and accurately applied in clinical practice. Furthermore, there are limited data on the topographical accuracy of FDT perimetry.

We conducted a prospective clinic based study comparing FDT perimetry with Humphrey 24-2 (H24-2) perimetry in a consecutive series of patients who were attending our glaucoma clinic. We determined the number of missed points on the FDT perimetry that optimised the sensitivity and specificity of the test in screening mode and determined the ability of FDT perimetry to localise the location and depth of a scotoma.

\section{Methods}

All patients involved in the study were attending the glaucoma service at the Stoke Mandeville eye unit, as either a review or a new consultation. Informed consent was obtained from each patient.

One hundred and ten consecutive patients underwent FDT testing in the screening mode, followed by H24-2 threshold testing of both eyes. After field testing all patients received an ophthalmic examination, including intraocular pressure measurement and evaluation of the optic discs, and were assigned a diagnosis based on the clinical and full threshold perimetric data. Legally blind eyes, and eyes with retinal pathology capable of producing a nerve fibre bundle defect, were excluded and to avoid confounding factors between the eyes, only the data from the right eye were analysed. Ninety nine eyes were included in the study and the information derived from the FDT perimetry was compared with the Humphrey field data using linear regression analysis and was analysed to determine the sensitivity and specificity of the FDT perimetry compared with the H24-2.

\section{Results}

There were 54 females and 45 males with an overall average age of 65.6 (SD 12.4) years in the study. Forty five eyes had open angle glaucoma, 36 eyes were classified as glaucoma suspect, three eyes had narrow angle glaucoma, and 15 eyes were normal.

The correlation between the total number of missed points on the FDT test and the Humphrey mean deviation (MD) score was $r$ $=0.79(\mathrm{p}<0.001) \quad($ Fig 1$)$. The correlation between the total number of missed points on the FDT perimetry and the Humphrey corrected pattern standard deviation (CPSD) score was not as strong as the correlation with the MD, but was still highly significant $(r$ $=0.56, \mathrm{p}<0.001)$. There were 24 eyes tested 


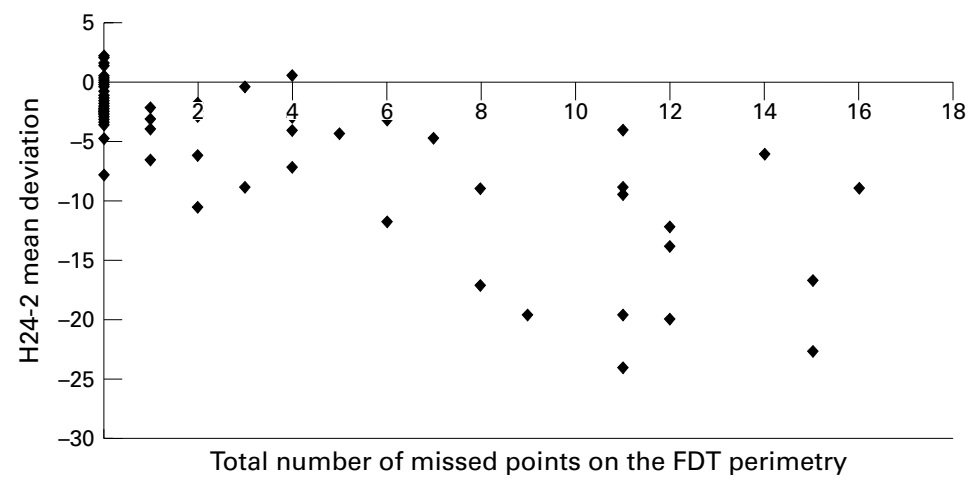

Figure 1 Scatter plot of Humphrey mean deviation compared with the total number of missed points on the frequency doubling technology test. The correlation is highly significant, $r=0.79, p<0.001$.

Table 1 Frequency doubling technology test compared with Humphrey hemifield test

\begin{tabular}{|c|c|c|c|c|c|c|}
\hline \multirow[b]{2}{*}{ Hemifield test } & \multicolumn{6}{|c|}{ Number of missed points on the FDT } \\
\hline & 0 misses & $\geqslant 1$ miss & $0-1$ misses & $\geqslant 2$ misses & 0-2 misses & $\geqslant 3$ misses \\
\hline Normal & 49 & 6 & 51 & 4 & 52 & 3 \\
\hline Abnormal & 7 & 25 & 11 & 21 & 13 & 19 \\
\hline Other* & 5 & 7 & 8 & 4 & 9 & 3 \\
\hline
\end{tabular}

^Borderline abnormal sensitivity.

$(12 \%)$ with more than five fixation losses on the H24-2, if these were excluded from the analysis the correlation between the $\mathrm{MD}$ and the total number of points missed on the FDT test was virtually unchanged $(r=0.76)$.

To estimate the optimal sensitivity and specificity of the FDT we divided the eyes into two groups based on the total number of missed points on the FDT test and compared them with the results of the Humphrey hemifield test as an indicator of a glaucomatous field defect (Table 1). Based on these data, using a criterion of at least one missed point on the FDT test as indicating abnormality, then the sensitivity was $78.1 \%(25 / 32)$ and the specificity was $89.1 \%$ (49/55). If two or more missed points on the FDT test were considered as the criterion for abnormality then the specificity marginally improved and the sensitivity was reduced to $65.6 \% .(21 / 32)$ (Table 1) If the Humphrey CPSD score was used as an indicator of an abnormal field instead of the hemifield test, and an abnormal test was defined as a CPSD probability of less than $5 \%$ and at least one missed point on the FDT test was considered to be abnormal, the sensitivity was $74.8 \%$ and the specificity was $89.1 \%$.

If glaucoma was defined as an abnormal hemifield test in the presence of a mean deviation of less than $6 \mathrm{~dB}$ and at least one missed point was considered as abnormal on the FDT test, then the sensitivity increased to $94.2 \%$ and the specificity was $89.3 \%$.

The average time to perform the FDT test was 57 (24) seconds per eye compared with an average time of over 12 minutes per eye on the Humphrey.

To determine whether the FDT test was accurately localising the scotomata we compared the total number of missed points on the FDT test in each quadrant with the total number of missed points on the mean deviation plot in the corresponding quadrant on the
H24-2 and found a strong correlation for each quadrant (superonasal quadrant $r=0.65$, superotemporal quadrant $r=0.56$, inferotemporal quadrant $r=0.60$, and inferonasal quadrant $r=0.65)$.

To determine whether the FDT test was accurately determining the depth of a scotoma we assigned a score to the abnormal points in the H24-2 total deviation plot, such that a location missed at the $\mathrm{p}<5 \%$ level was worth one point, a location missed at the $\mathrm{p}<2 \%$ level was worth two points, and the $\mathrm{p}<1 \%$ and $\mathrm{p}<0.5 \%$ scored 3 and 4 points respectively. Similarly, we assigned a score to the missed sectors on the FDT test such that mild, moderate, and severe defects were worth one, two, and three points respectively. We applied this scoring system to the points missed in the superonasal quadrant on both the $\mathrm{H} 24-2$ and the FDT perimetry and found a strong correlation $(r=0.68, \mathrm{p}<0.001)$.

\section{Discussion}

FDT is novel, rapid method of visual field testing which has had promising preliminary reports. ${ }^{8-16}$ After many years of experience now, the Humphrey field data can generally be interpreted in a meaningful and clinically relevant manner; however, the FDT data are currently difficult to interpret. To determine whether the FDT is producing topographically accurate information and to help interpret the data, we have compared the performance of FDT perimetry with the full threshold $\mathrm{H} 24-2$ in a clinical setting.

The strong correlation between the number of missed points on the FDT perimetry and the Humphrey perimetry has been reported by others ${ }^{815}$ and implies that counting the number of defects on the FDT may be a method of determining the presence and severity of glaucoma.

The optimal sensitivity and specificity of the FDT perimetry was $78.1 \%$ and $89.1 \%$ respectively, when an abnormal FDT test was defined as at least one missed point of any severity. Quigley reported a sensitivity of $91 \%$ and specificity of $94 \%$ when two or more abnormal points on the FDT represented an abnormality; however, the sensitivity and specificity if at least one abnormal point on the FDT test represented abnormality was not reported. ${ }^{8}$ Patel et al recently reported the use of an FDT scoring system based on the depth and location of the defect which was designed to aid interpretation of the FDT data. ${ }^{15}$ However, if an abnormal hemifield test was used to define glaucoma and the scoring system was replaced with the simpler criterion of at least one missed point on the FDT perimetry as defining abnormality then the sensitivity and specificity were only marginally reduced. ${ }^{15}$

The FDT was accurately able to determine the location of a field defect and the qualitative data concerning the depth of a scotoma were also consistent with the Humphrey data. This is important because it implies that with more experience it may be possible to use FDT data to determine if a known visual field defect is progressing. 
In conclusion, we have shown that FDT perimetry in the screening mode in a clinical setting performs favourably compared with the $\mathrm{H} 24-2$. It is a rapid, highly specific, and reasonably sensitive test, which can be considered abnormal when at least one missed point is present. The sensitivity in detecting moderate to severe glaucoma is over $90 \%$ without loss of specificity and it accurately reports the location and depth of a scotoma.

1 Kelly DH. Frequency doubling in visual responses. $\mathcal{f} O p t$ Soc Am A 1966;56:1628-33.

2 Maddess T, Henry GH. Performance of nonlinear visua units in ocular hypertension and glaucoma. Clin Vis $\mathrm{Sci}$ 1992;7:371-83.

3 Quigley HA, Dunkelberger GR, Baginski TA, et al. Chronic human glaucoma causes selectively greater loss of large human glaucoma causes selectively greater loss

4 Vickers JC, Shumer R, Podos S, et al. Selective vulnerability of neurochemically coded subpopulation of retinal neurons in a mourochemically coded subpopulation of retinal neurons

5 Maddess T, Goldberg I, Dobinson J, et al. Clinical trials of the frequency doubled illusion as an indicator of glaucoma. (ARVO Abstracts.) Invest Ophthalmol Vis Sci 1995;36 S335.

6 Maddess T, Severt WL. Testing for glaucoma with the frequency-doubling illusion in the whole, macula and eccentric visual fields. Aust NZ f Ophthalmol 1999;27:194-6.
7 Johnson CA, Cioffi GA, Van Buskirk EM. Frequency doubling technology perimetry using a $24-2$ stimulus presentation pattern. Optom Vis Sci 1999;76:571-81

8 Quigley HA. Identification of glaucoma-related visual field abnormality with the screening protocol of frequency doubling technology. Am f Ophthalmol 1998;125:819-29.

9 Brusini P, Busatto P. Frequency doubling perimetry in glaucoma early diagnosis. Acta Ophthalmol Scand Suppl 1998;227:23-4.

10 Chauhan BC, Johnson CA. Test-retest variability of frequency-doubling perimetry and conventional perimetry in glaucoma patients and normal subjects. Invest Ophthalmol Vis Sci 1999;40:648-56.

11 Yamada N, Chen PP, Mills RP, et al. Screening for glaucoma with frequency-doubling technology and Damato campimetry. Arch Ophthalmol 1999;117:1479-84.

12 Johnson CA, Samuels SJ. Screening for glaucomatous visual field loss with frequency-doubling perimetry. Invest Ophthalmol Vis Sci 1997;38:413-25.

13 Sponsel WE, Arango S, Trigo Y, et al. Clinical classification of glaucomatous visual field loss by freqeuncy doubling perimetry. Am f Ophthalmol 1998;125:830-6.

14 Cello KE, Nelson-Quigg JM, Johnson CA. Frequency doubling technology perimetry for detection of glaucomatous visual field loss. Am f Ophthalmol 2000;129:314-22.

15 Patel SC, Friedman DS, Varadkar P, et al. Algorithm for interpreting the results of frequency doubling perimetry. Am f Ophthalmol 2000;129:323-7.

16 Burnstein Y, Ellish NJ, Magbalon M, et al. Comparison of frequency doubling perimetry with Humphrey visual field analysis in a glaucoma practice. Am $\mathcal{f}$ Ophthalmol 2000;129:328-33. $<t w ; 4$ 